\title{
Cramer-Rao Lower Bound for Semiblind Channel Estimation with respect to Coded and Uncoded Finite-Alphabet Signals
}

\author{
Ansgar Scherb, Volker Kühn and Karl-Dirk Kammeyer \\ Department of Communications Engineering \\ University of Bremen, Otto-Hahn-Allee, D-28359 Bremen, Germany \\ Email:\{scherb, kuehn, kammeyer\}@ant.uni-bremen.de
}

\begin{abstract}
Within this paper we derive the Cramer-Rao lower bound (CRLB) for semiblind channel estimation with respect to coded or uncoded finite alphabet source signals in finite impulse response systems. Since the obtained solution incorporates a high dimensional integral, which can only be solved numerically, we approximate the CRLB for low and high signal to noise ratio (SNR). We also examine the SNR range where the CRLB crosses over from the low to the high SNR approximation. It will be shown that the crossover range depends on the modulation index and code characteristics. Combining the approximations for high and low SNR and estimating the crossover range yields an overall approximation, which can easily be calculated also for more complex scenarios. It will be shown, that the approximation meets the true CRLB very well.
\end{abstract}

\section{INTRODUCTION}

Methods for channel estimation can be classified as pilotdata aided, semiblind and completely blind. Usually, the source signal of a communication link consists of a training part, which contains symbols commonly known at the transmitter and the receiver, and a part bearing the information. Pilot-data aided channel estimation methods exploit solely the training part, whereas semiblind methods additionally consider the part of the unknown information signal. Completely blind methods do not require any training, but suffer from a phase ambiguity.

In order to evaluate methods for the estimation of FIRchannels, a theoretical lower bound limiting the estimation performance is of particular interest. The Cramer-Rao lower bound (CRLB) is a useful means, since it gives the minimum variance for an unbiased estimator. Considering pilot aided channel estimation the CRLB as well as a minimum variance unbiased (MVU) estimator attaining this bound is well known [1]. In case of completely blind channel estimation a reasonable CLRB can not be stated, since the channel can only be resolved up to an unknown complex factor and the remaining phase ambiguity is insufficiently modelled by the CRLB.

In this contribution we will focus on the CRLB of semiblind channel estimation methods, where at least one source symbol is specified to be a pilot. Carvalho and Slock have examined the CRLB for the semiblind multichannel case, where the channel and signal parameters of a continuous alphabet source were jointly estimated [2]. Sadler et al. have used an extended CRLB, where the finite alphabet (FA) character of the source signals is modelled as constraints [3]. The lower bound derived in [3] is exactly the same as if the complete source signals were a-priori known at the receiver. This is a reasonable result, but it will be shown that the CRLB at low SNR can be determined more accurately. Furthermore, this result does not provide insight into the impact of the modulation index on estimation variance.

This paper is organized as follows. In Section II the system model is presented and some quantities are defined. In Section III the CRLB for semiblind channel estimation with respect to coded and uncoded finite-alphabet signals is derived and an approximation of the CRLB is presented. The proposed approximation is verified by an example in Section IV. Finally, the results are concluded in Section V. Some detailed derivations of Section III were put into the Appendix.

\section{DATA MODEL AND NOTATION}

Consider a binary data sequence of $M$ bits denoted by $\mathbf{b}=$ $[b(1), \cdots, b(M)]^{T}, b(m) \in\{0,1\}$. Each data word is assigned to a distinct representation $\mathbf{s}=[s(1), \cdots, s(K)]^{T} \in \mathcal{A}$ in the $K$ dimensional signal space, where $s(k)$ is an element of a finite alphabet, e.g. PSK or QAM, and $\mathcal{A}=\left\{\mathbf{s}_{1}, \cdots, \mathbf{s}_{2^{M}}\right\}$ is the set of cardinality $|\mathcal{A}|=2^{M}$ comprising all realizations of $\mathbf{s}$. Note that $\mathbf{s}$ may include pilot symbols as well as channel coding. If all data words $\mathbf{b}$ are equally probable, the a-priori the probability $p\left(\mathbf{s}_{i}\right)$ for any $\mathbf{s}_{i} \in \mathcal{A}$ is $1 /|\mathcal{A}|$. The symbols $\mathbf{s}$ are transmitted over a frequency selective channel of order $L$. We assume that the interval between two consecutive data blocks is filled by a sufficiently large number of zero symbols, such that no inter symbol interference between two consecutive blocks occurs. Collecting $K+L$ samples in the vector $\mathbf{r}$ at symbol rate, the channel output is given by

$$
\mathbf{r}=\mathbf{S h}+\mathbf{n}
$$

where $\mathbf{S}$ is the $(L+K \times L+1)$ convolution matrix of $\mathbf{s}$ defined by

$$
[\mathbf{S}]_{\nu, \mu}=\left\{\begin{array}{lll}
s(\nu-\mu+1) & : & 0 \leq \nu-\mu<K \\
0 & : & \text { else }
\end{array} .\right.
$$

The vector $\mathbf{h}=\left[h_{0}, \cdots, h_{L}\right]^{T}$ contains the channel gains $h_{l} \in \mathbb{C}$ including transmit and receive filter and $\mathbf{n}=$ $[n(1), \cdots, n(L+K)]^{T}$ is white gaussian noise with power $\sigma_{n}^{2}$. 
Without loss of generality we assume that the mean power of one symbol is $\mathrm{E}\left\{|s(k)|^{2}\right\}=1$.

We call $\mathbf{S}_{i} \mathbf{h}$ the $i$-th receive hypothesis, since it would be the observation at the receiver under the assumption that the word $\mathbf{s}_{i} \in \mathcal{A}$ was transmitted over a noiseless channel $\mathbf{h}$. The distance between the $i$-th and $j$-th receive hypothesis is defined as

$$
\delta_{i, j}=\left\|\left(\mathbf{S}_{i}-\mathbf{S}_{j}\right) \mathbf{h}\right\|
$$

and the minimum distance of a signal mapping configuration is defined as

$$
\delta_{\min }=\min _{\mathbf{s}_{i}, \mathbf{s}_{j} \in \mathcal{A}}\left\|\left(\mathbf{S}_{i}-\mathbf{S}_{j}\right) \mathbf{h}\right\|
$$

where $i \neq j$.

In order to obtain a validated statement by the CRLB, it has to be guaranteed that the estimation problem has an unique solution for all $\mathbf{s}_{i} \in \mathcal{A}$. For this reason a necessary condition is that the minimum distance $\delta_{\min }$ is larger than zero, i.e.

$$
\delta_{\min }>0 \text {. }
$$

\section{CRAMER-RAO LOWER BOUND}

In this section we will derive an expression for the CRLB. Since the derived equation can be only resolved numerically for very short block lengths, we present approximations for the high and low SNR regime. Furthermore, the SNR range, where the expression for high SNR changes to the expression low SNR, will be estimated. Here, we will focus on the main results, whereas some mathematical derivations were put into the Appendix.

\section{A. Derivation of the CRLB}

The CRLB can be stated as follows: Let $\hat{\mathbf{h}}$ be an unbiased estimator of the channel parameter $\mathbf{h}$ and $\mathbf{C}_{\hat{\mathbf{h}}}=\mathrm{E}\{(\mathbf{h}-\hat{\mathbf{h}})(\mathbf{h}-$ $\left.\hat{\mathbf{h}})^{H}\right\}$ be the covariance of the estimator. Then the covariance matrix of any unbiased estimator satisfies

$$
\mathbf{C}_{\hat{\mathbf{h}}}-\mathbf{F}^{-1} \geq \mathbf{0}
$$

where " $\geq 0$ " stands for "positive semidefinite". $\mathbf{F}$ is the Fisher-information matrix (FIM) [1] given by the expectation

$$
\mathbf{F}=-\mathrm{E}\left\{\frac{\partial^{2} \log p(\mathbf{r} \mid \mathbf{h})}{\partial \mathbf{h} \partial \mathbf{h}^{H}}\right\},
$$

where

$$
p(\mathbf{r} \mid \mathbf{h})=\sum_{\mathbf{s}_{i} \in \mathcal{A}} p\left(\mathbf{r} \mid \mathbf{s}_{i}, \mathbf{h}\right) p\left(\mathbf{s}_{i}\right)
$$

and

$$
p\left(\mathbf{r} \mid \mathbf{s}_{i}, \mathbf{h}\right)=\frac{1}{\left(\pi \sigma_{n}^{2}\right)^{L+K}} \exp \left(-\left\|\mathbf{r}-\mathbf{S}_{i} \mathbf{h}\right\|^{2} / \sigma_{n}^{2}\right) .
$$

The minimum mean squared error (MSE) obtained by any unbiased estimator can directly be derived from the inverse FIM by $\mathrm{MSE}_{\min }=\operatorname{trace}\left(\mathbf{F}^{-1}\right)$. Defining

$$
\mathbf{d}_{i}=\frac{\mathbf{r}-\mathbf{S}_{i} \mathbf{h}}{\sigma_{n}}
$$

and inserting (8) and (9) into (7) the desired FIM is given by

$$
\mathbf{F}=\frac{1}{|\mathcal{A}| \sigma_{n}^{2}} \int_{\mathbb{C}^{L+K}} \frac{\sum_{\mathbf{s}_{i}, \mathbf{s}_{j} \in \mathcal{A}} \mathbf{S}_{i}^{H} \mathbf{d}_{i} \mathbf{d}_{j}^{H} \mathbf{S}_{j} p\left(\mathbf{r} \mid \mathbf{s}_{i}, \mathbf{h}\right) p\left(\mathbf{r} \mid \mathbf{s}_{j}, \mathbf{h}\right)}{\sum_{\mathbf{s}_{i} \in \mathcal{A}} p\left(\mathbf{r} \mid \mathbf{s}_{i}, \mathbf{h}\right)} d \mathbf{r} .
$$

The integration of this expression can only be done numerically and the expense of calculating $\mathbf{F}$ is approximately proportional to $|\mathcal{A}|^{2} / 2$. Since $|\mathcal{A}|$ may become large e.g. for higher modulation order or long sequences, in most cases this expression does not provide an intuitive insight of the CRLB.

B. Approximation of the CRLB for the high and low SNR regime

As derived in Appendix A, an approximation for high SNR can be obtained by

$$
\mathbf{F}_{\text {high }}=\frac{1}{|\mathcal{A}| \sigma^{2}} \sum_{\mathbf{s}_{i} \in \mathcal{A}} \mathbf{S}_{i}^{H} \mathbf{S}_{i},
$$

whereas in Appendix B the low SNR approximation

$$
\mathbf{F}_{\text {low }}=\frac{1}{|\mathcal{A}|^{2} \sigma^{2}} \sum_{\mathbf{s}_{i}, \mathbf{s}_{j} \in \mathcal{A}} \mathbf{S}_{i}^{H} \mathbf{S}_{j}
$$

will be derived. Thus, the minimum MSE of any unbiased estimator is within the range $\operatorname{trace}\left(\mathbf{F}_{\text {high }}^{-1}\right) \leq \mathrm{MSE}_{\min } \leq$ $\operatorname{trace}\left(\mathbf{F}_{\text {low }}^{-1}\right)$. For most signal mapping configurations (12) and (13) can easily be calculated. Let e.g. $\mathbf{S}$ consist of a training and an information bearing part by $\mathbf{S}=\mathbf{S}_{\mathrm{T}}+\mathbf{S}_{\mathrm{I}}$, where $s_{\mathrm{I}}(k)$ is zero mean, $\mathrm{E}\left\{\mathbf{s}_{\mathrm{I}}^{H} \mathbf{s}_{\mathrm{I}}=\tilde{K}\right\}$, and

$$
\mathrm{E}\left\{s_{\mathrm{I}}(k) s_{\mathrm{I}}(k+\kappa)\right\}=\left\{\begin{array}{lll}
1 & : & \kappa=0 \\
0 & : & \text { else }
\end{array} .\right.
$$

The zero mean property and (14) hold for conventional symbol mappings (as e.g. PSK and QAM) in connection with most linear channel codes.

In this particular case the approximations of the FIM according to (12) and (13) are given by

$$
\begin{aligned}
\mathbf{F}_{\text {high }} & =\frac{1}{|\mathcal{A}| \sigma^{2}} \sum_{\mathbf{s}_{i} \in \mathcal{A}}\left(\mathbf{S}_{\mathrm{T}}^{H}+\mathbf{S}_{\mathrm{I}, i}^{H}\right)\left(\mathbf{S}_{\mathrm{T}}+\mathbf{S}_{\mathrm{I}, i}\right) \\
& =\frac{1}{\sigma_{n}^{2}} \mathbf{S}_{\mathrm{T}}^{H} \mathbf{S}_{\mathrm{T}}+\underbrace{\frac{1}{|\mathcal{A}| \sigma_{n}^{2}} \sum_{\mathbf{s}_{i} \in \mathcal{A}} \mathbf{S}_{\mathrm{I}, i}^{H} \mathbf{S}_{\mathrm{I}, i}}_{\tilde{K} \mathbf{I}} .
\end{aligned}
$$

and

$$
\begin{aligned}
\mathbf{F}_{\text {low }} & =\frac{1}{\sigma_{n}^{2}}(\mathbf{S}_{\mathrm{T}}^{H}+\underbrace{\frac{1}{|\mathcal{A}|} \sum_{\mathbf{s}_{i} \in \mathcal{A}} \mathbf{S}_{\mathrm{I}, i}^{H}}_{=\mathbf{0}})(\mathbf{S}_{\mathrm{T}}+\underbrace{\frac{1}{|\mathcal{A}|} \sum_{\mathbf{s}_{j} \in \mathcal{A}} \mathbf{S}_{\mathrm{I}, j}}_{=\mathbf{0}}) \\
& =\frac{1}{\sigma_{n}^{2}} \mathbf{S}_{\mathrm{T}}^{H} \mathbf{S}_{\mathrm{T}}
\end{aligned}
$$

It is remarkable that $\mathbf{F}_{\text {low }}$ depends only on the pilot part $\mathbf{s}_{\mathrm{T}}$. Therefore, in the low SNR regime the semiblind CRLB is nearly identical to the CRLB of a pilot aided channel estimator. 
In contrast, $\mathbf{F}_{\text {high }}$ takes all available symbols into account. Hence, in the high SNR regime the semiblind CRLB is close to the case of perfectly knowing the values of each symbol at the estimator.

\section{The crossover SNR range}

As shown in Appendix $\mathrm{C}$, the crossover SNR range, where the FIM changes from (12) to (13), can be approximately determined with respect to the squared distance noise ratio $\delta_{\min }^{2} / \sigma_{n}^{2}$. If the noise power is within the range

$$
\delta_{\min } / 4.87<\sigma_{n}^{2}<\delta_{\min } / 0.08,
$$

the CRLB varies between both extremes. Otherwise, (12) and (13) provide an accurate match of the true CRLB characteristics.

Please note that in coding theory one possible metric to evaluate a code is the minimum Hamming distance $d_{\text {min }}$. The distance $\delta_{\min }$ can be understood as the multivariate signal space counterpart of $d_{\min }$, where the connection between both is approximately (large block length and high code rates) given by

$$
\delta_{\min } \approx d_{\min } a_{\min }\|\mathbf{h}\|
$$

and $a_{\min }$ is the minimum symbol distance of the symbol alphabet, e.g. PSK or QAM.

\section{EXAMPLE}

In order to demonstrate our CRLB approximation we consider an uncoded example $\mathcal{A}_{u}=\{\mathbf{s}=$ $\left.\left[d_{1}, \cdots, d_{4}, c(1), \cdots, c(4)\right]^{T}\right\}$, where $d_{1}, \cdots, d_{4} \in\{-1,+1\}$ are $D$ pilots fixed for all $\mathbf{s} \in \mathcal{A}_{u}$ and $c(m) \in\{-1,+1\}$ are BPSK symbols bearing the information (Figure 1). Additionally, the channel coded example $\mathcal{A}_{c}=\{\mathbf{s}=$ $\left.\left[d_{1}, c(1), \cdots, c(7)\right]^{T}\right\}$ was examined, where $d_{1}$ is one pilot and $c(1), \cdots, c(7)$ are channel encoded BPSK symbols obtained by a $(7,4)$-Hamming code (Figure 2). Note that the degree of redundancy is in both cases the same, as $\mathbf{s}$ is always of dimension 8 bearing the same amount of information. We chose such a small block length $K$, since for these scenarios it is possible to approximate the true CRLB numerically. The channel impulse response was $\mathbf{h}=[0.5,1 / \sqrt{2}, 0.5]^{T}$ and the minimum hypothesis distances were consequently $\delta_{\min , u}=2$ and $\delta_{\min , c}=2.828$. As it can be seen in Fig. 1 the upper limit $\operatorname{trace}\left(\mathbf{F}_{\text {low }}^{-1}\right) \approx(L+1) \sigma_{n}^{2} / D$ is given by the minimum MSE obtained if only the training part of $\mathbf{s}$ were used for channel estimation, whereas the lower limit $\operatorname{trace}\left(\mathbf{F}_{\text {high }}^{-1}\right) \approx(L+1) \sigma_{n}^{2} / K$ is given by the minimum MSE obtained if all symbols of $\mathrm{s}$ were pilots. Since the part of training is in the first example larger than in the second, the upper limits of both schemes differ significantly, whereas the lower limit is identical. The estimated crossover region is underlaid by grey color. It can be observed that in the channel coded example this region is slightly shifted to a lower SNR range. Hence, it can be assumed that the quality of semiblind channel estimation methods can be improved by appropriate code design. Furthermore, Figs. 1 and 2 show that the proposed approximation meets quite well the true CRLB.

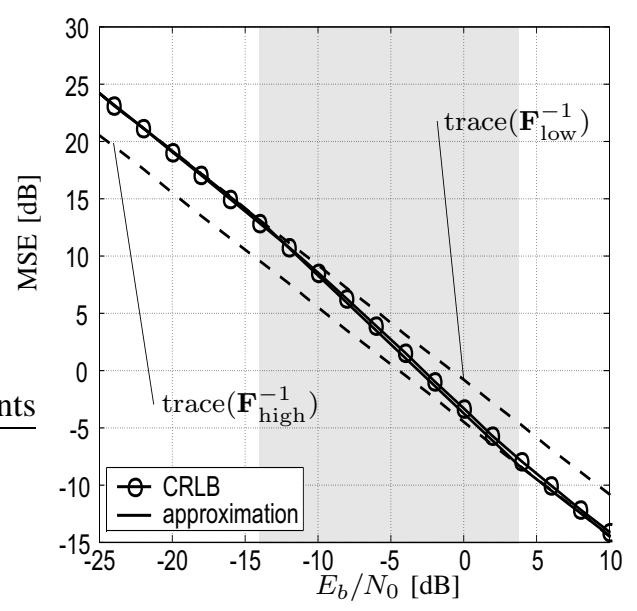

Fig. 1. CRLB and its approximation for example I

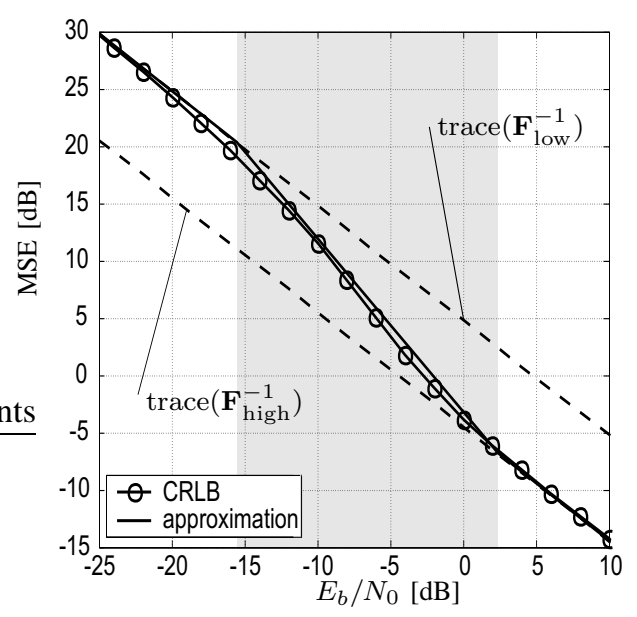

Fig. 2. CRLB and its approximation for example II

\section{CONCLUSiON}

In this paper we derived the CRLB for semiblind channel estimation of frequency selective channels with respect to channel coding and modulation degree. Since the derived expression can only be reasonably tackled for very short block lengths, we introduced approximations of the FIM at high and low SNR and gave an estimation for the crossover SNR range. The position of the crossover SNR range is governed by coding properties namely the minimum Hamming distance and the modulation degree. We have shown that in case of zero mean symbols and linear channel coding the derived approximations can be easily calculated. In an example it is demonstrated that the approximation meets well the true CRLB.

\section{APPENDIX}

\section{A. Approximation for high SNR}

In order to characterize the behavior of the probability density function (PDF) $p\left(\mathbf{r} \mid \mathbf{s}_{i}, \mathbf{h}\right)$ for any $\mathbf{s}_{i} \in \mathcal{A}$, we define the radius $\rho$ so that for an arbitrary $\mathbf{s}_{i} \in \mathcal{A}$ the approximation

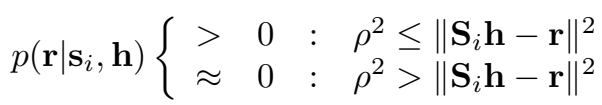




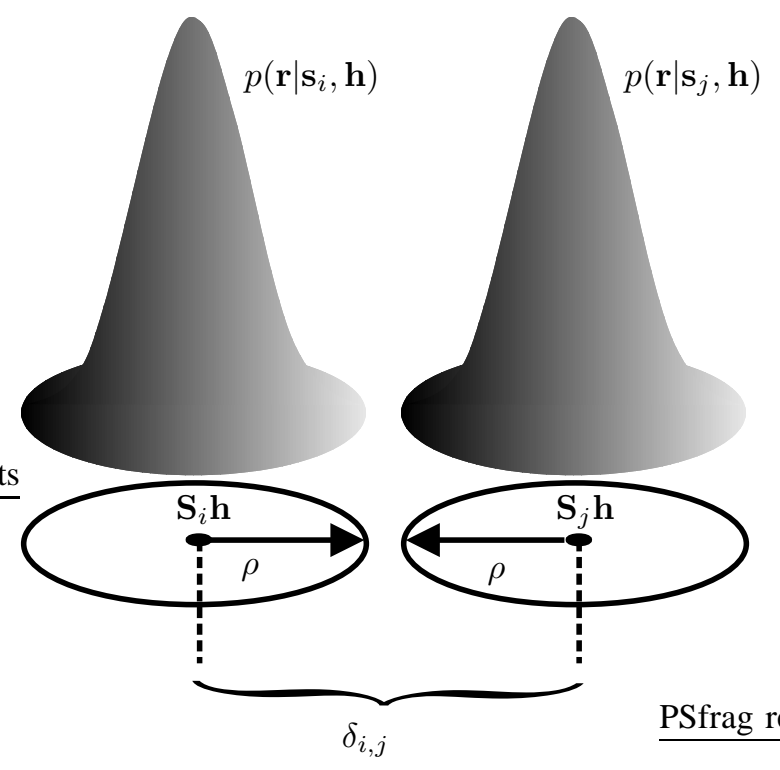

Fig. 3. Illustration of the overlap regarding the signifi cant nonzero parts of $p\left(\mathbf{r} \mid \mathbf{s}_{i}, \mathbf{h}\right)$ and $p\left(\mathbf{r} \mid \mathbf{s}_{j}, \mathbf{h}\right)$ at high SNR

holds. In other words, the normal distribution $p\left(\mathbf{r} \mid \mathbf{s}_{i}, \mathbf{h}\right)$ significantly differs from zero, if $\mathbf{r}$ is within a $\rho$-sized sphere around the center $\mathbf{S}_{i} \mathbf{h}$ and is approximately zero else. Please note that the radius $\rho$ depends on the noise power $\sigma_{n}^{2}$ and is identical for any $p\left(\mathbf{r} \mid \mathbf{s}_{i}, \mathbf{h}\right), \mathbf{s}_{i} \in \mathcal{A}$.

For high SNR the radius $\rho$ becomes small and, thus, the distance between an arbitrary pair of receive hypotheses is larger than twice the radius $\rho$, i.e.

$$
2 \rho \leq \delta_{\min }
$$

In other words, none of any pair $p\left(\mathbf{r} \mid \mathbf{s}_{i}, \mathbf{h}\right)$ and $p\left(\mathbf{r} \mid \mathbf{s}_{j}, \mathbf{h}\right)$, $i \neq j$, commonly differ significantly from zero at any point $\mathbf{r}$ (Fig. 3).

Hence, for $i \neq j$ the product

$$
p\left(\mathbf{r} \mid \mathbf{s}_{i}, \mathbf{h}\right) p\left(\mathbf{r} \mid \mathbf{s}_{j}, \mathbf{h}\right) \approx 0
$$

over the whole range $\mathbf{r} \in \mathbb{C}^{K+L}$. Therefore, in the high SNR regime (11) can be simplified to

$$
\mathbf{F}_{\text {high }} \approx \frac{1}{|\mathcal{A}| \sigma_{n}^{2}} \int_{\mathbf{r} \in \mathbb{C}^{L+K}} \frac{\sum_{\mathbf{s}_{i}, \in \mathcal{A}} \mathbf{S}_{i}^{H} \mathbf{d}_{i} \mathbf{d}_{i}^{H} \mathbf{S}_{i}\left[p\left(\mathbf{r} \mid \mathbf{s}_{i}, \mathbf{h}\right)\right]^{2}}{\sum_{\mathbf{s}_{i} \in \mathcal{A}} p\left(\mathbf{r} \mid \mathbf{s}_{i}, \mathbf{h}\right)} d \mathbf{r}
$$

Due to (19), within the sphere $\rho^{2} \leq\left\|\mathbf{S}_{j} \mathbf{h}-\mathbf{r}\right\|^{2}$ the sums in the numerator and the denominator of (22) are dominated by the corresponding PDF $p\left(\mathbf{r} \mid \mathbf{s}_{j}, \mathbf{h}\right)$. Thus,

$$
\sum_{\mathbf{s}_{i} \in \mathcal{A}} \mathbf{S}_{i}^{H} \mathbf{d}_{i} \mathbf{d}_{i}^{H} \mathbf{S}_{i}\left[p\left(\mathbf{r} \mid \mathbf{s}_{i}, \mathbf{h}\right)\right]^{2} \approx \mathbf{S}_{j}^{H} \mathbf{d}_{j} \mathbf{d}_{j}^{H} \mathbf{S}_{j}\left[p\left(\mathbf{r} \mid \mathbf{s}_{j}, \mathbf{h}\right)\right]^{2}
$$

and

$$
\sum_{\mathbf{s}_{i} \in \mathcal{A}} p\left(\mathbf{r} \mid \mathbf{s}_{i}, \mathbf{h}\right) \approx p\left(\mathbf{r} \mid \mathbf{s}_{j}, \mathbf{h}\right) .
$$

Hence, (22) can be further simplified by splitting the integral into $|\mathcal{A}|$ disjoint pieces covering a $\rho$-sized sphere around the centers $\mathbf{S}_{i} \mathbf{h}, \mathbf{s}_{i} \in \mathcal{A}$ and replacing the numerator and the denominator of (22) by the r.h.s. of (23) and (24), respectively:

$$
\begin{aligned}
\mathbf{F}_{\text {high }} & =\frac{1}{|\mathcal{A}| \sigma_{n}^{2}} \sum_{\mathbf{s}_{i} \in \mathcal{A}} \int_{\rho^{2} \leq\left\|\mathbf{S}_{i} \mathbf{h}-\mathbf{r}\right\|^{2}} \mathbf{S}_{i}^{H} \mathbf{d}_{i} \mathbf{d}_{i}^{H} \mathbf{S}_{i} p\left(\mathbf{r} \mid \mathbf{s}_{i}, \mathbf{h}\right) d \mathbf{r} \\
& =\frac{1}{|\mathcal{A}| \sigma_{n}^{2}} \sum_{\mathbf{s}_{i} \in \mathcal{A}} \mathrm{E}\left\{\mathbf{S}_{i}^{H} \mathbf{d}_{i} \mathbf{d}_{i}^{H} \mathbf{S}_{i} \mid \mathbf{s}_{i}, \mathbf{h}\right\} \\
& =\frac{1}{|\mathcal{A}| \sigma_{n}^{2}} \sum_{\mathbf{s}_{i} \in \mathcal{A}} \mathbf{S}_{i}^{H} \mathbf{S}_{i}
\end{aligned}
$$

\section{B. Approximation for low SNR}

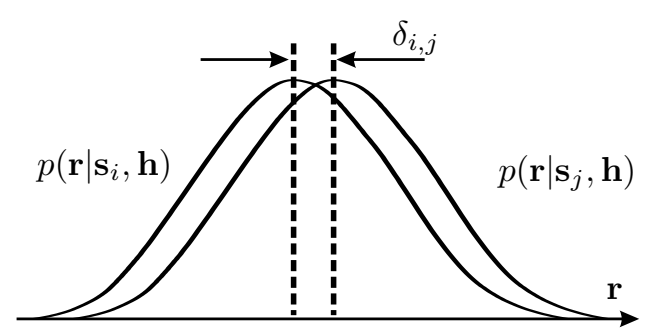

Fig. 4. Illustration of the overlap regarding the signifi cant nonzero parts of $p\left(\mathbf{r} \mid \mathbf{s}_{i}, \mathbf{h}\right)$ and $p\left(\mathbf{r} \mid \mathbf{s}_{j}, \mathbf{h}\right)$ at low SNR

In contrast to the high SNR approximation, the low SNR approximation is based on the assumption

$$
\sigma_{n} \gg \delta_{i, j}
$$

for any admissible pair $(i, j)$. Hence, $\mathbf{d}_{i}$ can be approximated by

$$
\begin{aligned}
\mathbf{d}_{i} & =\frac{\left(\mathbf{S}_{j}-\mathbf{S}_{i}\right) \mathbf{h}+\mathbf{n}}{\sigma_{n}} \\
& \approx \frac{\mathbf{n}}{\sigma_{n}}
\end{aligned}
$$

From (27) follows

$$
p\left(\mathbf{r} \mid \mathbf{s}_{i}, \mathbf{h}\right) \approx \frac{1}{\left(\pi \sigma_{n}^{2}\right)^{L+K}} \exp \left(-\|\mathbf{n}\|^{2} / \sigma_{n}^{2}\right)=p(\mathbf{n})
$$

for any $\mathbf{s}_{i} \in \mathcal{A}$. Thus, the low SNR approximation states that all PDFs of this type are nearly identical (Fig. 4). Replacing the r.h.s. of (27) and (28) in (11) yields

$$
\begin{aligned}
\mathbf{F}_{\text {low }} & \approx \frac{1}{|\mathcal{A}|^{2} \sigma_{n}^{2}} \sum_{\mathbf{s}_{i}, \mathbf{s}_{j} \in \mathcal{A}} \int_{\mathbb{C}^{L+K}} \mathbf{S}_{i}^{H} \mathbf{n} \mathbf{n}^{H} \mathbf{S}_{j} p(\mathbf{n}) d \mathbf{n} \\
& =\frac{1}{|\mathcal{A}|^{2} \sigma^{2}} \sum_{\mathbf{s}_{i}, \mathbf{s}_{j} \in \mathcal{A}} \mathbf{S}_{i}^{H} \mathbf{S}_{j}
\end{aligned}
$$

\section{Determining the crossover SNR range}

Recall that the approximations $\mathbf{F}_{\text {high }}$ and $\mathbf{F}_{\text {low }}$ are essentially based on assumptions regarding the overlap of the significant nonzero parts of different PDFs $p\left(\mathbf{r} \mid \mathbf{s}_{i}, \mathbf{h}\right), \mathbf{s}_{i} \in \mathcal{A}$. In the following the degree of overlap of the significant nonzero parts of two PDFs is termed as mutual correlation. 
Let $p_{+}(\mathbf{x}) \sim \mathcal{N}\left(\boldsymbol{\mu}, \sigma_{n}^{2} \mathbf{I}_{N}\right)$ and $p_{-}(\mathbf{x}) \sim \mathcal{N}\left(-\boldsymbol{\mu}, \sigma_{n}^{2} \mathbf{I}_{N}\right)$ be two complex $N$-variate normal distributions with identical variance and $\delta=\|2 \boldsymbol{\mu}\|$ is the distance between both means. The mutual correlation of both distributions depends on the squared distance variance ratio $\delta^{2} / \sigma_{n}^{2}$. The function

$$
\begin{aligned}
Q_{\delta}\left(\sigma_{n}^{2}\right) & =\int_{\mathbb{C}^{N}}\left(p_{+}(\mathbf{x}) \cdot p_{-}(\mathbf{x})\right)^{1 / 2} d \mathbf{x} \\
& =\exp \left(\frac{\delta^{2}}{4 \sigma_{n}^{2}}\right)
\end{aligned}
$$

becomes one for $\sigma_{n}^{2} \rightarrow \infty$ and zero for $\sigma_{n}^{2} \rightarrow 0$. Thus, $Q_{\delta}\left(\sigma_{n}^{2}\right)$ can be understood as a measurement of the mutual correlation. In the context of the system presented in Section II, the worst case distance between two PDFs is $\delta_{\text {min }}$. For this reason the crossover SNR range can be approximately located, where $Q_{\delta_{\min }}\left(\sigma_{n}^{2}\right)$ is between $5 \%$ and $95 \%$ of its maximum, i.e.

$$
0.05<Q_{\delta_{\min }}\left(\sigma_{n}^{2}\right)<0.95 .
$$

Replacing the r.h.s. of (30) in (31) delivers a crossover SNR range estimation according to (17).

\section{REFERENCES}

[1] S.M. Kay. Fundamentals of Statistical Signal Processing, Volume I: Estimation Theory. Prentice Hall PTR, 1993.

[2] E. D. Carvalho and D. T. Slock. Cramer Rao bounds for semiblind, blind and training sequence based channel estimation. In 1st IEEE signal processing workshop on signal processing advances in wireless communications (SPAWC), Paris, France, 1997.

[3] B. M. Sadler, R.J. Kozick, and T. Moore. Bounds on bearing and symbol estimation with side information. IEEE Trans. on Signal Processing, 49:822 - 834, 2001. 